\title{
BMJ Open Induction of labour in case of premature rupture of membranes at term with an unfavourable cervix: protocol for a randomised controlled trial comparing double balloon catheter (+oxytocin) and vaginal prostaglandin (RUBAPRO) treatments
}

\author{
Eric Devillard, ${ }^{1}$ Amélie Delabaere, ${ }^{1,2}$ Marion Rouzaire, ${ }^{1}$ Bruno Pereira, ${ }^{3}$ \\ Marie Accoceberry, ${ }^{1}$ Céline Houlle, ${ }^{1}$ Lydie Dejou-Bouillet, ${ }^{1}$ Pamela Bouchet, ${ }^{1}$ \\ Denis Gallot ${ }^{1,2}$
}

To cite: Devillard E, Delabaere A, Rouzaire M, et al. Induction of labour in case of premature rupture of membranes at term with an unfavourable cervix: protocol for a randomised controlled trial comparing double balloon catheter (+oxytocin) and vaginal prostaglandin (RUBAPR0) treatments. BMJ Open 2019;9:e026090. doi:10.1136/ bmjopen-2018-026090

- Prepublication history and additional material for this paper are available online. To view these files, please visit the journal online (http://dx.doi org/10.1136/bmjopen-2018026090 )

Received 21 August 2018 Revised 7 March 2019 Accepted 9 May 2019

Check for updates

(c) Author(s) (or their employer(s)) 2019. Re-use permitted under CC BY-NC. No commercial re-use. See rights and permissions. Published by BMJ.

For numbered affiliations see end of article.

Correspondence to

Prof. Denis Gallot;

dgallot@chu-clermontferrand.fr

\section{ABSTRACT}

Introduction Premature rupture of membranes (PROM) occurs at term in $8 \%$ of pregnancies. Several studies have demonstrated that the risk of chorioamnionitis and neonatal sepsis increases with duration of PROM. Decreasing the time interval between PROM and delivery is associated with lower rates of maternal infections. In case of an unfavourable cervix, the use of prostaglandin for cervical maturation demonstrates some advantages over oxytocin. The use of double balloon catheter in reduction of PROM duration has not been evaluated in the literature.

Methods and analysis We are conducting a prospective, monocentric, randomised clinical trial on pregnant women with an unfavourable cervix showing PROM at term (RUBAPR0). After 12-24 hours of PROM, women are randomly assigned to one group treated with a double balloon catheter for 12 hours, with oxytocin administered after 6 hours or to the control group treated with 24 hours of vaginal prostaglandin followed by oxytocin infusion alone. Patients $(n=80)$ are randomised at a 1:1 ratio with stratification on parity. The inclusion criteria are a Bishop score of $<6$, cephalic presentation at term and confirmed PROM. Women with suspected chorioamnionitis; group B streptococcus (GBS) carrier; a history of caesarean delivery or any contraindication for vaginal delivery are excluded. The time from induction to delivery is the primary outcome. Secondary outcomes were mode of delivery, maternofetal morbidity and the effect of parity on strategies for reduction of PROM duration. To sufficiently demonstrate a difference (10 hours) between groupswith a statistical power of $90 \%$ and a two-tailed $\alpha$ of $5 \%-40$ patients per group will be required.

Ethics and dissemination Written informed consent is required from participants. National Ethics Committee approval was obtained in August 2017. The results will be published in a peer-reviewed journal and presented at relevant conferences. Access to raw data will be available only to members of the research team.
Strengths and limitation of this study

RUBAPRO is the first randomised parallel-group trial to compare the use of double balloon catheter and oxytocin with pharmacological cervical ripening with vaginal prostaglandin among women with premature rupture of membranes (PROM) at term.

- This method could represent a viable treatment strategy with shortening of time interval from induction to delivery in case of PROM at term with an unfavourable cervix.

- Physicians and patients cannot be blinded to treatment.

- Caesarean delivery may reduce the induction to delivery without being clinically beneficial because of the surgical procedure performed.

- In order to compensate this limitation, the data 'duration of induction, labour and caesarean procedure in the event of an adverse outcome' will be replaced by the maximum value obtained in the study for duration of induction and labour in a patient with vaginal delivery.

Trial registration number NCT03310333.

\section{INTRODUCTION}

Premature rupture of membranes (PROM) at term is defined as rupture of membranes at least 1 hour before the onset of uterine contractions at a gestational age of 37 weeks or more. It complicates $8 \%$ of pregnancies. ${ }^{1}$ This diagnosis is associated with a risk of chorioamnionitis, which increases with duration of PROM. ${ }^{2}$ 
Several studies show that latency beyond 24 hours increases the incidence of chorioamnionitis and neonatal sepsis. ${ }^{23}$ Spontaneous labour occurs in $60 \%-67 \%$ of these patients within 24 hours. ${ }^{2}$ If no labour occurs, labour induction seems to be the optimal strategy for women with PROM at term.

In 2009, the American College of Obstetricians and Gynecologists recommended using oxytocin for induction of labour in case of PROM at term, even if the cervix is unfavourable. ${ }^{5}$ Several other labour-inducing options are available, including prostaglandin, misoprostol and Foley catheters; however, none of these approaches demonstrates superiority over oxytocin in inducing labour, even when the cervix is unfavourable. ${ }^{67}$ Several studies have evaluated the combination of oxytocin and mechanical ripening devices, as oxytocin alone has not been shown to affect the risk of chorioamnionitis. ${ }^{8-11}$ However, a recent study by Mackeen $e t$ al demonstrated that groups treated with oxytocin and Foley catheters suffered higher rates of chorioamnionitis than groups treated with oxytocin alone, with no greater reduction in the time interval from induction to delivery. ${ }^{12}$ It is noteworthy that patients with group B streptococcus (GBS) colonisation were enrolled in this study and antibioprophylaxis was administered only in case of positive GBS and suspected intra-amniotic infection.

The study TERMPROM found that vaginal prostaglandin help to reduce latency and shorten total labour time compared with expectative management in unfavourable cervix cases. ${ }^{3}$ Vaginal prostaglandin treatment does not increase rates of caesarean extraction or maternal or fetal morbidity; however, this pharmacological ripening method carries the risk of uterine hyperstimulation, which can adversely affect fetal heart rate (FHR). ${ }^{13} 14$

Induction of labour by mechanical device is accepted in non-PROM cases, although mechanical treatment does not show any advantage over vaginal prostaglandin regarding rates of chorioamnionitis, endometritis and neonatal infection. ${ }^{15}$ Some studies have compared single and double balloon catheters, yet no differences in effects were observed. ${ }^{16}{ }^{17}$ Mechanical devices do seem to present lower haemorrhaging risks than pharmacological approaches, but not to a statistically significant degree. $^{918}$

This study evaluates the combined use of double balloon catheters provided by Cook Medical Europe (Ireland; reference: J-CRBS-184000) and oxytocin, as compared with treatment with vaginal prostaglandin. Our review of the literature, conducted on PubMed and ClinicalTrial. gov, uncovered no prior research on the use of this type of double balloon catheter in case of PROM at term, with or without administration of oxytocin.

We hypothesise that mechanical ripening using double balloon catheters and oxytocin will reduce the time between the onset of ripening and delivery. This method could represent a viable treatment strategy with reduced risk of uterine hyperstimulation in case of PROM at term with an unfavourable cervix.

\section{METHODS AND ANALYSIS}

\section{Study design}

We are conducting a randomised controlled clinical trial at the University Hospital of Clermont-Ferrand, France on 80 patients with unfavourable cervices showing PROM at term without spontaneous labour. The trial was designed to investigate whether treatment using a double balloon catheter and oxytocin may improve the induction of labour in case of PROM at term.

The study RUBAPRO is a prospective, controlled, randomised, therapeutic and monocentric clinical trial with two parallel arms, comparing induction via double balloon catheter and oxytocin with vaginal prostaglandin.

This article respect items from the WHO Trial Registration Data Set and Standard Protocol Items: Recommendations for Interventional Trials checklist, provided in the online supplementary appendix.

\section{Study population}

To be eligible for the trial, inclusion criteria include the following conditions:

- $\geq 18$ years of age.

- Pregnant and at term ( $\geq 37$ weeks of gestation; gestational age estimated by ultrasonography between 11 and 13 (+6) weeks of gestation).

- Carrying a live singleton fetus in cephalic presentation.

- Show PROM through either clinical diagnosis or biological detection of Insulin like Growth Factor Binding Protein (IGFBP)-1 in cervicovaginal secretions (via the ActimProm test), and not enter spontaneous labour within 12-24 hours of PROM.

- Bishop score of $<6$;

- Consent to the study after being provided clear and appropriate information.

- Affiliated with French healthcare.

Exclusion criteria include the following conditions:

- Positive detection of GBS or (Streptococcus agalactiae) on any vaginal or urinary sample during the current or a previous pregnancy.

- Contraindication for vaginal delivery (praevia placenta).

- Presence of meconium in the amniotic fluid at time of recruitment.

- Fever at time of recruitment.

- Suspicion of chorioamnionitis based on Newton's criteria.

- Fetal growth restriction $<3$ rd percentile with Doppler abnormalities.

- Severe congenital fetal anomalies.

- Uterine scarring or history of caesarean delivery.

- Abnormal pre-induction FHR tracing.

- Suspicion of genital herpes infection.

- Known HIV seropositivity.

- Contraindication for ripening via double balloon catheters, vaginal prostaglandin or oxytocin.

Only women meeting all inclusion criteria and presenting no exclusion criteria can be included in the trial. 
Confirmed PROM at term

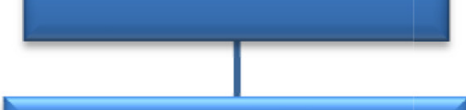

Streptococcus B neg. or unknown: expectative for 12-24 hours
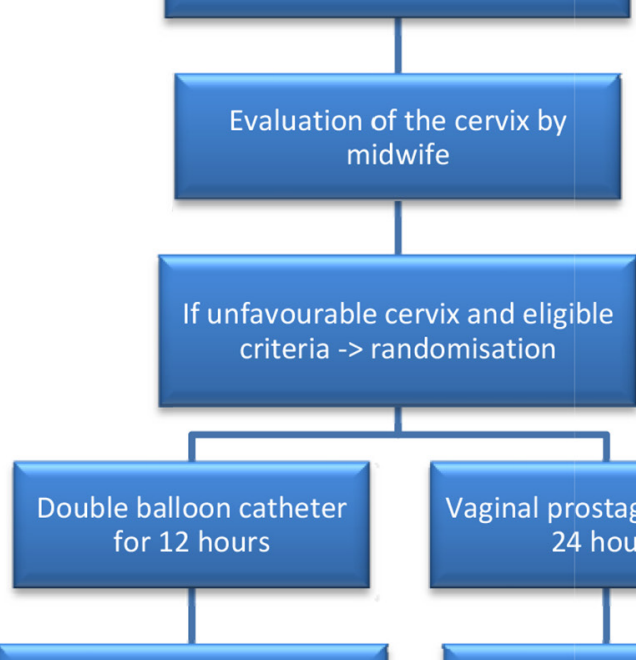

Adjunction of oxytocin after six hours with catheter
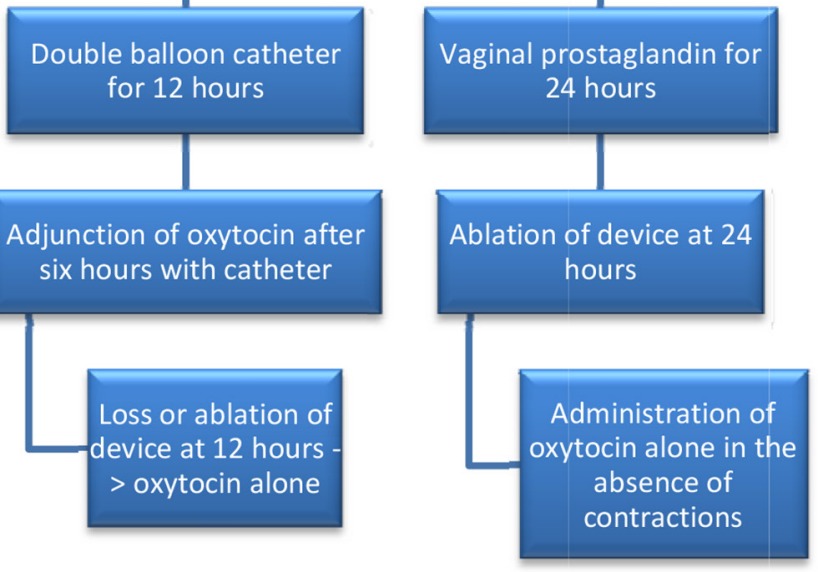

Figure 1 Flow chart describing the main stages of the study. PROM, premature rupture of membrane.

\section{Study protocol \\ Recruitment}

During pregnancy, patients are often informed by obstetricians and midwives of potentially significant symptoms such as membranes rupture. When a pregnant woman is admitted with this condition, fetal cardiotocography and a gynaecological examination are performed, and a diagnosis is made either clinically (by observing amniotic fluid via the cervix, spontaneously or during a coughing test) or using a biological test (ActimProm). If the rupture is confirmed, the woman is admitted. Vaginal, urinary and blood samples are taken. Patients showing contractions are accepted in the delivery room. In other cases, patients are kept 12-24 hours for expectant management.

Once the patient's eligibility has been determined, she received a clear verbal explanation and printed information about the study by obstetricians. Written consent is obtained from patients. The main stages of the study are described in figure 1.

A course of prophylactic antibiotics-amoxicillin, or clindamycin in case of penicillin allergy-is begun on recruitment and extends to the delivery to prevent chorioamnionitis.

\section{Randomisation}

Randomisation and data collection are conducted using Research Electronic Data Capture (REDCap) software. The randomisation algorithm requires confirmation of the presence of all inclusion criteria and the absence of exclusion criteria. Randomisation is carried out in random-sized blocks with stratification on parity to avoid prognostic imbalance between groups. The randomisation algorithm is known only by the data manager of REDCap, and not by any of the doctors.

\section{Intervention}

Participants are assigned to either the mechanical or the pharmacological cervical ripening group:

- Mechanical cervical ripening (Cook Cervical Ripening Balloon, Cook Medical Europe, Ireland, reference: J-CRBS-184000):

The catheter is placed according to the manufacturer's instructions. ${ }^{19}$ Each balloon is filled with $40-80 \mathrm{~mL}$ of saline solution, as tolerated by the patient. Expected volume is $80 \mathrm{~mL}$. No traction is realised on the catheter. The device remains in place for 12 hours. The FHR is monitored for $30 \mathrm{~min}$ before and 2 hours after placement.

Oxytocin is injected 6 hours after placement, with epidural anaesthesia as per patient's demand.

The cervix is controlled after removal of the catheter (H12), or earlier if the patient has severe pain or spontaneously expulses the device. Administration of oxytocin is continued according to uterine contractions after catheter loss or removal.

- Pharmacological cervical ripening (prostaglandin PGE2 (Propess), Ferring SAS, Gentilly, France):

A Propess vaginal system is inserted by a midwife, with or without a speculum. As in the catheter procedure, FHR is monitored $30 \mathrm{~min}$ before and 2 hours after placement.

The vaginal system is placed for a maximum of 24 hours. If it is expulsed in the first 12 hours and the patient has no contractions or still shows an unfavourable cervix, another vaginal system is placed for a maximum of another 24 hours.

After this first step, the procedure continues with intravenous oxytocin, which can be administered $30 \mathrm{~min}$ after removal of the vaginal system, with or without epidural anaesthesia as per the patient's request.

For this patient group, FHR is checked every 6 hours.

\section{Patient follow-up}

After the induction of labour, patient pain is assessed every 6 hours until device removal; clinical management is the same for both groups and follows the service protocol. Oxytocin is administered according to contractions and FHR, and the midwife monitors temperature, blood pressure and pain, as recommended. ${ }^{20}$ 
If the patient experiences an obstructed labour, the midwife must call the physician on duty. FHR abnormalities are interpreted according to revised FIGO classifications. ${ }^{21}$ The final delivery method (vaginal delivery or caesarean extraction) is decided by the physician on duty.

After delivery, the midwife takes samples from the placenta and newborn (gastric fluid, umbilicus, anus and ear canal).

After 2 hours of postdelivery supervision, the patient is taken back to her room. Data regarding potential maternofetal infections are recorded and used in the analysis. No additional visits after discharge are scheduled in the protocol.

\section{Blinding}

The nature of intervention makes it impossible to blind the physicians, midwives or patients.

\section{Objectives}

Primary objective

To demonstrate that labour induction via the Cook double balloon catheter combined with oxytocin treatment can reduce the time between induction of labour and delivery-as compared with vaginal prostaglandin treatment, which is the accepted recommendation-in case of PROM at term with an unfavourable cervix.

\section{Secondary objectives}

To compare maternofetal impacts of each induction strategy, and to study how parity affects each plan.

\section{Study end points}

Primary outcome

The primary outcome is the time between induction (the hour of placement of induction device) and delivery (the hour of birth).

\section{Secondary outcomes}

\section{Maternal evaluation}

Time between PROM and the beginning of induction; rate of delivery within the first 24 hours; duration of induction device placement; rate of spontaneous or assisted vaginal delivery; rate of caesarean section and indications; rate of postpartum haemorrhage (blood loss $>500 \mathrm{~mL}$ ); Bishop score or measure of dilatation on loss or removal of catheter; rate of balloon expulsion within 12 hours of placement; rate of oxytocin cessation due to suboptimal FHR; rate of balloon or vaginal prostaglandin ablation due to suboptimal FHR; rate of epidural analgesia; time taken to achieve active labour and full dilatation; pain levels at time of placement, every 6 hours and after the ablation; rate of uterine hyperstimulation (defined as $>6$ contractions/10 $\mathrm{min}$ over any $30 \mathrm{~min}$ period); rate of fever during labour; rate of chorioamnionitis based on Newton's criteria, rate of endometritis, rate of histological chorioamnionitis and positive bacterial culture.

\section{Neonatal morbidity}

Birth weight, rate of Apgar score $<7$ at $5 \mathrm{~min}$, rate of umbilical artery $\mathrm{pH}<7.15$, rate of neonatal intensive care unit admission, rate of maternal fetal infection.

\section{Patient and public involvement}

This study investigates patients' interests. The regional ethics committee is composed by patients' representatives who gave their agreement for the design of the protocol. By means of the committee, data about pain during placement of device and the ripening are recorded. After the ripening, safety profiles will be recorded by the following question: "for next pregnancy, if a ripening will be indicated, will you agree to have the same device of ripening?"

Patients are clearly informed of the objectives, course, benefits and risks of the study and can ask all their questions to the investigator before giving their consent. An informative flyer presenting the results will be sent to them at the end of the study at their request.

\section{Sample size calculation}

We determined sample size with the aim of highlighting a significant difference between randomised groups in the time between induction (the hour of placement of induction device) and delivery (hour of birth). According to data from our centre and a study by Mackeen $e t a l,{ }^{10}$ the SD of the primary outcome will be around 10 hours. To achieve a two-sided type I error of $5 \%$ with a statistical power of $90 \%$ and to highlight a clinical and relevant absolute difference of 9 hours, 26 patients per group are needed. However, to guarantee satisfactory statistical power for secondary outcomes, we propose to include 40 patients per group.

\section{Statistical analysis}

All analysis will be conducted with Stata (V.13, StataCorp, College Station, Texas, USA) and R (http://cran.rproject.org) software, in line with the International Conference on Harmonization Good Clinical Practice guidelines. A two-sided $p$ value of $<0.05$ will be used for statistical significance. Intention-to-treat analysis will be the primary analysis method. A per-protocol analysis will also be conducted to account for protocol deviations. Patients who withdraw consent will not be included in the analysis. Continuous variables will be presented as means and SD, or as medians and quartiles. Normality will be assessed by the Shapiro-Wilk test, and homoscedasticity by the Fisher-Snedecor test.

The comparison of the primary outcome between randomised groups will be performed using Student's t-test, or a non-parametric Mann-Whitney $U$ test if the assumptions of t-test will not met. Results will be reported as effect sizes with $95 \%$ CIs. Then, multivariable analyses will be conducted using linear regression models to account for possible confounding covariates, with models selected according to clinical relevance and stratification variables. Normality of residuals will be 
assessed using the Shapiro-Wilk test. If appropriate, a logarithmic transformation will be proposed to achieve normality of the primary outcome. The results will be expressed as regression coefficients with $95 \%$ CIs. In a secondary analysis, the primary outcome will be also treated as censored data associated with a favourable outcome (uncomplicated delivery, without caesarean section), so the estimation will be realised using the Kaplan-Meier method and comparison performed through a log-rank test. For multivariable analysis, Cox proportional hazards analysis will be carried out. Proportional hazard assumptions will be verified by applying the Schoenfeld test and plotting residuals. Results will be reported as HRs with $95 \%$ CIs.

Student's t-test or Mann-Whitney U test will be used for other quantitative parameters such as time from PROM to induction; duration of induction device placement; Bishop score; time taken to achieve active labour and full dilatation; birth weight and levels of neonatal arterial blood gases. Results will be reported as effect sizes with $95 \%$ CIs; $X^{2}$ test or Fisher's exact test will be used for categorical parameters, including rates of delivery within the first 24 hours; spontaneous or assisted vaginal delivery; caesarean section; postpartum haemorrhage; balloon expulsion within 12 hours of catheter placement; oxytocin cessation due to suboptimal FHR; balloon or vaginal prostaglandin ablation due to suboptimal FHR; epidural analgesia; uterine hyperstimulation; fever during labour; Apgar score $<7$ at 5 min; neonatal intensive care unit admission; maternal infection; chorioamnionitis based on Newton's criteria; endometritis and histological chorioamnionitis. Results will be expressed as absolute differences with $95 \%$ CIs. Then, multivariate analyses will be conducted using regression models: linear regression for quantitative outcomes and generalised linear regression for categorical outcomes. The results will be expressed, respectively, as regression coefficients and relative risks, with $95 \%$ CIs.

Concerning the study of the parameters collected longitudinally (in particular, evaluation of pain caused by the induction device on placement, every 6 hours and after ablation), mixed models will be used to take into account between and within patient variability (as random-effect). The following fixed effects will be analysed: randomisation group, time and interactions (timexgroup).

According to clinical relevance, European Medicines Agency and Consolidated Standards of Reporting Trials recommendations, subgroup analysis will be proposed after the study of subgroups $\times$ randomisation group interactions in regression models. Missing values will be noted and analysed (this is particularly relevant to the fact that performing a caesarean may hasten delivery without overall clinical benefit). More precisely, a sensitivity analysis will be performed and the nature of missing data will be evaluated to determine the role of randomness. If the frequency is $>5 \%$, additional studies will be performed using the multiple imputation methods.

\section{Data Monitoring Safety Committee}

A Data Monitoring Safety Committee (independent from the laboratory) will discuss the results of intermediate analysis. Committee meetings will cover blocks of 20 patients. The committee consists of two independent members who work in the University Hospital of Clermont-Ferrand (one a gynaecologist who does not work in obstetrics unit, one paediatrician), and two doctors of public health from Grenoble University Hospital (France).

The committee will examine all records concerning the transfer of participating patients or their newborn infants to intensive care or reanimation units. Intermediate safety analysis will be conducted concerning the rate of caesarean and chorioamnionitis. The analysis will be performed both with and without knowledge of what data correspond to which study group, in order to determine which is the exposed group. The study will be halted if the exposed group is the double balloon catheter group.

\section{Data storage and management}

Data storage and management are performed according to international guidelines relevant in French institutions. All data are recorded by a resident and a clinical research associate. The data are entered into the randomisation software REDCap ${ }^{22}$ and anonymised. Data quality control measures will include queries to identify outliers and missing data. Only the two above-mentioned staff members and the study principal investigator (PI) will have access to protected personal health information. After inclusion, a unique identifier will be stored in a locked cabinet. The PI will ensure that anonymity is preserved. The PI will have access to the final trial data set, as will a biostatistician.

An adverse event (AE) is defined as any untoward medical occurrence in a patient taking part in the study. A serious $\mathrm{AE}$ is defined as any untoward occurrence that requires hospitalisation or prolongation of existing hospitalisation; results in persistent or significant disability; is life-threatening or results in death or is otherwise considered medically significant by the investigator. $\mathrm{AE}$ will be recorded on an AE form. An assessment of the gravity, possible causality and expectedness event/reaction will be undertaken. Resolution of these events will be entered on the $\mathrm{AE}$ form.

\section{Ethics and dissemination}

The first patient inclusion was on 12 February 2018, and we hope to recruit a total of 80 patients within 18 months of the first recruitment.

The protocol respects the Declaration of Helsinki. This monocentric, randomised, parallel-group trial is registered at http://www.clinicaltrials.gov under the registration number NCT03310333. We will publish findings from this study in a peer-reviewed scientific journal and present results at national and international conferences, regardless of whether they are statistically significant.

The study is funded from the research budget of the University Hospital of Clermont-Ferrand, and receives no 
funding from double balloon catheter or vaginal prostaglandin insert manufacturers.

\section{DISCUSSION}

RUBAPRO is the first monocentric, open-label, parallel-group, randomised controlled trial to compare treatment using a double balloon catheter combined with oxytocin with treatment with vaginal prostaglandin in case of PROM at term with an unfavourable cervix.

Our objective is to shorten the time interval between the beginning of ripening and delivery, which could decrease the risk of chorioamnionitis and therefore of maternal or fetal infection.

If it is not shown to worsen maternofetal morbidity, the use of the Cook double balloon catheter in case of PROM may be authorised as a viable induction strategy. Further trials are also planned to explore the use of double balloon catheters in patients with colonisation of GBS.

Some studies have found a lower rate of caesarean section for uterine hyperstimulation when mechanical ripening devices are used instead of prostaglandin. We know caesarean is associated with extended hospitalisation, thromboembolic risk, postoperative wound infection and higher cost; regarding fetal morbidity, caesarean increases the risk for neonatal respiratory distress, admission to neonatal care unit and neonatal mortality.

If our hypothesis is confirmed, quicker ripening via double balloon catheter and oxytocin will decrease time spent in the delivery room, thus improving prospects for and reducing the cost of delivery.

Limitations of this study include the impossibility of blinding.

\section{CONCLUSIONS}

This trial is built to demonstrate a reduction of time between induction and delivery when a mechanical ripening device and oxytocin are used instead of a pharmacological approach in case of PROM at term with an unfavourable cervix. Results could support the use of double balloon catheters as a viable treatment for PROM.

\section{Author affiliations}

${ }^{1}$ Obstetrics and Gynaecology Department, Clermont-Ferrand University Hospital, Clermont-Ferrand, France

${ }^{2}$ Translational approach to epithelial injury and repair' team, Auvergne University, CNRS, Inserm, GReD, Clermont-Ferrand, France

${ }^{3}$ Biostatistics 1 Unit, Clermont-Ferrand University Hospital, Clermont-Ferrand, France

Acknowledgements The authors would like to thank data manager Patrick Lacarin and Nicolas Andant for their help during the trial preparation and Ferass Sendy for his help in proofreading the article.

Contributors ED, MR, DG developed the study concept, design and aims, designed data collection tools, analysed the data and drafted and revised the paper. ED, MR monitored data collection throughout the study. AD, MA, CH, LD-B provided substantial contributions to the conception and design of the work and co-investigators for patients' inclusion. BP provided statistical expertise in clinical trial design, aided in the analysis plan and analysis of the results and revised the paper. $E D, A D, M R, B P, M A, C H, L D-B, P B, D G$ contributed to refinement of the study protocol and approved the final manuscript.

Funding The authors have not declared a specific grant for this research from any funding agency in the public, commercial or not-for-profit sectors.

Competing interests None declared.

Patient consent for publication Not required.

Ethics approval Authorisations from the University Hospital, the regional ethics committee (CPP Sud Méditerranée IV, France) and the French National Agency for Medicines and Health Products Safety have been obtained since November 2017.

Provenance and peer review Not commissioned; externally peer reviewed.

Open access This is an open access article distributed in accordance with the Creative Commons Attribution Non Commercial (CC BY-NC 4.0) license, which permits others to distribute, remix, adapt, build upon this work non-commercially, and license their derivative works on different terms, provided the original work is properly cited, appropriate credit is given, any changes made indicated, and the use is non-commercial. See: http://creativecommons.org/licenses/by-nc/4.0/.

\section{REFERENCES}

1. Gunn GC, Mishell DR, Morton DG. Premature rupture of the fetal membranes. A review. Am J Obstet Gynecol 1970;106:469-83.

2. Hannah ME, Ohlsson A, Farine D, et al. Induction of labor compared with expectant management for prelabor rupture of the membranes at term. TERMPROM Study Group. N Engl J Med 1996;334:1005-10.

3. Seaward PG, Hannah ME, Myhr TL, et al. International Multicentre Term Prelabor Rupture of Membranes Study: evaluation of predictors of clinical chorioamnionitis and postpartum fever in patients with prelabor rupture of membranes at term. Am J Obstet Gynecol 1997;177:1024-9.

4. Huret C, Pereira B, Collange V, et al. Prédiction de l'entrée en travail sous 24 heures en cas de rupture des membranes à terme. Gynécologie Obstétrique Fertilité \& Sénologie 2017;45:348-52.

5. ACOG Committee on Practice Bulletins -- Obstetrics. ACOG Practice Bulletin No. 107: Induction of labor. Obstet Gynecol 2009;114:386-97.

6. Tan BP, Hannah ME. Prostaglandins versus oxytocin for prelabour rupture of membranes at term. Cochrane Database Syst Rev 2000;2:CD000159.

7. Middleton P, Shepherd E, Flenady V, et al. Planned early birth versus expectant management (waiting) for prelabour rupture of membranes at term (37 weeks or more). Cochrane Database Syst Rev 2017;04:CD005302.

8. Cabrera IB, Quiñones JN, Durie D, et al. Use of intracervical balloons and chorioamnionitis in term premature rupture of membranes. $J$ Matern Fetal Neonatal Med 2016;29:967-71.

9. Amorosa JMH, Stone J, Factor $\mathrm{SH}$, et al. A randomized trial of Foley Bulb for Labor Induction in Premature Rupture of Membranes in Nulliparas (FLIP). Am J Obstet Gynecol 2017;217:360.e1-360.e7.

10. Mackeen AD, Walker L, Ruhstaller K, et al. Foley catheter vs prostaglandin as ripening agent in pregnant women with premature rupture of membranes. J Am Osteopath Assoc 2014;114:686-92.

11. Wolff K, Swahn ML, Westgren M. Balloon catheter for induction of labor in nulliparous women with prelabor rupture of the membranes at term. A preliminary report. Gynecol Obstet Invest 1998;46:1-4.

12. Mackeen AD, Durie DE, Lin M, et al. Foley Plus Oxytocin Compared With Oxytocin for Induction After Membrane Rupture: A Randomized Controlled Trial. Obstet Gynecol 2018;131:4-11.

13. Vaknin Z, Kurzweil Y, Sherman D. Foley catheter balloon vs locally applied prostaglandins for cervical ripening and labor induction: a systematic review and metaanalysis. Am J Obstet Gynecol 2010;203:418-29.

14. Thomas J, Fairclough A, Kavanagh J, et al. Vaginal prostaglandin (PGE2 and PGF2a) for induction of labour at term. Cochrane Database Syst Rev 2014;6:CD003101.

15. McMaster K, Sanchez-Ramos L, Kaunitz AM. Evaluation of a Transcervical Foley Catheter as a Source of Infection: A Systematic Review and Meta-analysis. Obstet Gynecol 2015;126:539-51.

16. Yang F, Huang S, Long Y, et al. Double-balloon versus single-balloon catheter for cervical ripening and labor induction: A systematic review and meta-analysis. J Obstet Gynaecol Res 2018;44:27-34.

17. Lajusticia H, Martínez-Domínguez SJ, Pérez-Roncero GR, et al. Project THO and SA (HOUSSAY). Single versus double-balloon catheters for the induction of labour of singleton pregnancies: a meta-analysis of randomized and quasi-randomized controlled trials. Arch Gynecol Obstet 2018:1-12. 
18. Kruit $\mathrm{H}$, Tihtonen $\mathrm{K}$, Raudaskoski $\mathrm{T}$, et al. Foley Catheter or Oral Misoprostol for Induction of Labor in Women with Term Premature Rupture of Membranes: A Randomized Multicenter Trial. Am J Perinatol 2016;33:866-72.

19. Cook Medical. Cervical ripening balloon with stylet. $2016 \mathrm{https} / / /$ www.cookmedical.com/data/resources/RH-D25085-FRCA-F_M3_ 1463771416666.pdf

20. HAS. Recommandation de bonne pratique. Avril. Déclenchement artificiel du travail à partir de 37 semaines d'aménorrhée. 2008 https://www.has-sante.fr/portail/jcms/c 666473/fr/declenchementartificiel-du-travail-a-partir-de-37-semaines-d-amenorrhee

21. Ayres-de-Campos D, Spong CY, Chandraharan E. FIGO Intrapartum Fetal Monitoring Expert Consensus Panel. FIGO consensus guidelines on intrapartum fetal monitoring: Cardiotocography. Int $J$ Gynaecol Obstet 2015;131:13-24.

22. Harris PA, Taylor R, Thielke R, et al. Research electronic data capture (REDCap)--a metadata-driven methodology and workflow process for providing translational research informatics support. $J$ Biomed Inform 2009;42:377-81. 